\title{
A comparative analysis of the compression characteristics of a thermoplastic polyurethane 3D printed in four infill patterns for comfort applications
}

\author{
Susan Erica Nace \\ School of Mechanical and Materials Engineering, University College Dublin, Dublin, Ireland and \\ SeatTech Posture and Mobility Services, Enable Ireland, Dublin, Ireland \\ fohn Tiernan \\ SeatTech Posture and Mobility Services, Enable Ireland, Dublin, Ireland, and \\ Donal Holland and Aisling Ni Annaidh \\ School of Mechanical and Materials Engineering, University College Dublin, Dublin, Ireland
}

\begin{abstract}
Purpose - Most support surfaces in comfort applications and sporting equipment are made from pressure-relieving foam such as viscoelastic polyurethane. However, for some users, foam is not the best material as it acts as a thermal insulator and it may not offer adequate postural support. The additive manufacturing of such surfaces and equipment may alleviate these issues, but material and design investigation is needed to optimize the printing parameters for use in pressure relief applications. This study aims to assess the ability of an additive manufactured flexible polymer to perform similarly to a viscoelastic foam for use in comfort applications.

Design/methodology/approach - Three-dimensional (3D) printed samples of thermoplastic polyurethane (TPU) are tested in uniaxial compression with four different infill patterns and varying infill percentage. The behaviours of the samples are compared to a viscoelastic polyurethane foam used in various comfort applications.

Findings - Results indicate that TPU experiences an increase in strength with an increasing infill percentage. Findings from the study suggest that infill pattern impacts the compressive response of 3D printed material, with two-dimensional patterns inducing an elasto-plastic buckling of the cell walls in TPU depending on infill percentage. Such buckling may not be a beneficial property for comfort applications. Based on the results, the authors suggest printing from TPU with a low-density 3D infill, such as $5 \%$ gyroid.

Originality/value - Several common infill patterns are characterised in compression in this work, suggesting the importance of infill choices when 3D printing end-use products and design for manufacturing.
\end{abstract}

Keywords 3D printing, Thermoplastic polymers, Compression loading, Stress (materials), Infill patterns

Paper type Research paper

\section{Introduction}

According to an analysis conducted by Grand View Research Inc. in 2017, the size of the global pressure relief devices market will rise from US\$2.53bn in 2015 to US $\$ 4.14$ bn in 2025 (Grand View Research Inc., 2017). This rise is expected due to an increasing geriatric and limited-mobility population who are at an increased risk of pressure injury development, which pressure relief devices are designed to prevent or help heal. Viscoelastic polyurethane foams (VPFs) are commonly used in various comfort cushioning applications related to pressure relief, including hospital or long-term care beds, wheelchair

The current issue and full text archive of this journal is available on Emerald Insight at: https://www.emerald.com/insight/1355-2546.htm

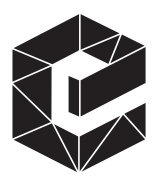

Rapid Prototyping Journal

27/11 (2021) 24-36

Emerald Publishing Limited [ISSN 1355-2546]

[DOI $10.1108 /$ RPJ-07-2020-0155] seating, office furniture, shoe sole inserts and aircraft and vehicle seating. VPFs are commonly used in these applications

(c) Susan Erica Nace, John Tiernan, Donal Holland and Aisling Ni Annaidh. Published by Emerald Publishing Limited. This article is published under the Creative Commons Attribution (CC BY 4.0) licence. Anyone may reproduce, distribute, translate and create derivative works of this article (for both commercial and non-commercial purposes), subject to full attribution to the original publication and authors. The full terms of this licence may be seen at http://creativecommons.org/licences/by/4.0/legalcode

This work has been funded by the Irish Research Council and Enable Ireland through the Employment Based Postgraduate Programme (grant number EBP/2017/498) and University College Dublin's School of Mechanical and Materials Engineering.

Conflict of interest statement: The authors have no conflict of interest in the research to declare.

Received 6 July 2020

Revised 25 January 2021

12 April 2021

Accepted 14 April 2021 
due to their ability to conform to the user's body shape with increased usage time, increasing the surface area over which body pressure is distributed, thus lowering peak pressures experienced by the user.

Other key factors contributing to increased risk of pressure injury development are shear and friction at the surface interface, local temperature and local humidity at the usersurface interface (Lachenbruch, 2005; Lachenbruch et al., 2015; Schwartz et al., 2018). Foams thermally insulate users from their surrounding environment over the surface area of the foam. At times, this insulation can lead to an increase in localised moisture and temperature that heightens the risk of pressure injury development in that region (Jan et al., 2013), which can present a severe health and financial risk (Stroupe et al., 2011; Dealey et al., 2012). The heightened temperature can also lead to increased discomfort in environments such as offices, home bedding, personal vehicles and in the use of protective clothing and gear for sports (Finn et al., 2012; Kumar et al., 2015; Dotti et al., 2016; Califano et al., 2017). The use of materials other than VPF and the development of new support surface designs may allow for better control of the microclimate at the support surface-user interface while still offering pressure and shear relief to the tissues at risk of pressure injury or discomfort. With the development of three-dimensional (3D) printing in clinical settings, the appropriateness of $3 \mathrm{D}$ printed cushioning materials should be examined, especially qualifying their ability to support weightbearing loads encountered in comfort applications.

\subsection{Fused filament fabrication}

Additive manufacturing through fused filament fabrication (FFF), commonly referred to as $3 \mathrm{D}$ printing, is a relatively inexpensive manufacturing process that builds a part up in thin layers. To attain flexible, elastic or energy-absorbent parts made using FFF, materials such as thermoplastic elastomers, specifically thermoplastic polyurethanes (TPUs), are more effective to use as they are inherently flexible. Bulk TPU material has been modelled by Qi and Boyce (2004) as a hyperelastic, rubbery network with a viscoelastic-plastic element. When 3D printed, its behaviour in compression is similar to that of elastomeric foams (Avalle et al., 2001). The effect of 3D printing parameters on the elasticity and energyabsorption of TPU has been investigated recently (Bates et al., 2016, 2018; Rossiter et al., 2019) and multi-material prints including TPU are on the rise for use in biomedical applications (Tsai et al., 2017; Verstraete et al., 2018; Lin et al., 2019). Whether TPU is suitable for use as an alternative to VPF in comfort applications is yet to be determined and is the goal of the current study. Herein TPU specimens of varying infill percentages and infill patterns are tested in compression and compared to a soft fire-retardant grade SunMate foam (Dynamic Systems, Inc., USA). This VPF is commonly used in comfort applications, including hospital bedding, Formula One headrests, custom prosthetics and wheelchair seating and aircraft and motorcycle seating.

\subsection{Infill patterns}

The internal infill percentage and infill pattern of the object are usually defined in the slicing software and the patterns available to the manufacturer vary depending on the slicing software
Figure 1 Examples of 2D infill patterns in the Ultimaker Cura slicer software version 4.5

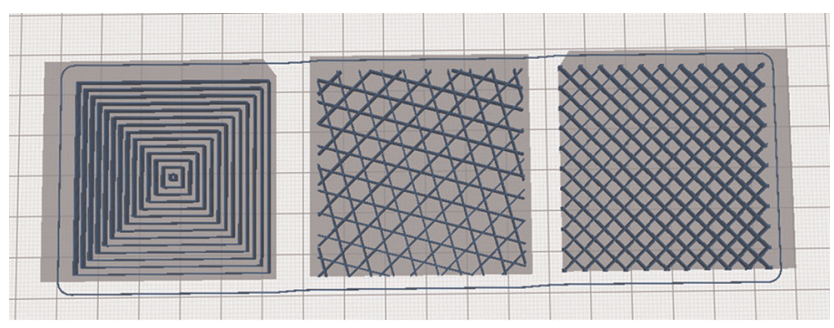

Note: Pictured from left to right are concentric infill, tri-hexagonal infill, and grid infill in a cuboid

used. Two-dimensional (2D) structures are the most common type of FFF infill patterns. From layer to layer in the normal direction to the printer build plate, the inner area of a 2D infill pattern does not change. Examples of $2 \mathrm{D}$ infill patterns are shown in Figure 1. 2D patterns cause anisotropy in the mechanical properties of the printed part, making print direction an important design factor when using such patterns (Kozior and Kundera, 2017).

Some slicing software also offers $3 \mathrm{D}$ infill patterns, such as those shown in Figure 2. A 3D infill pattern has cells with 3D built from sliced flat layers that vary in the direction normal to the printer build plate. Some 3D infill patterns are made up of triply periodic minimal surfaces (TPMS), such as the gyroid infill shown in Figure 2. TPMS are commonly found in natural structures, do not self-intersect and are isotropic (DomínguezRodríguez et al., 2018; Podroužek et al., 2019).

When $3 \mathrm{D}$ printing end-use parts, being able to predict how printing parameters such as infill pattern and infill percentage will affect the performance of the part is necessary. This need has motivated research into the behaviour of $3 \mathrm{D}$ printed

Figure 2 Examples of 3D infill patterns in Ultimaker Cura slicer software version 4.5

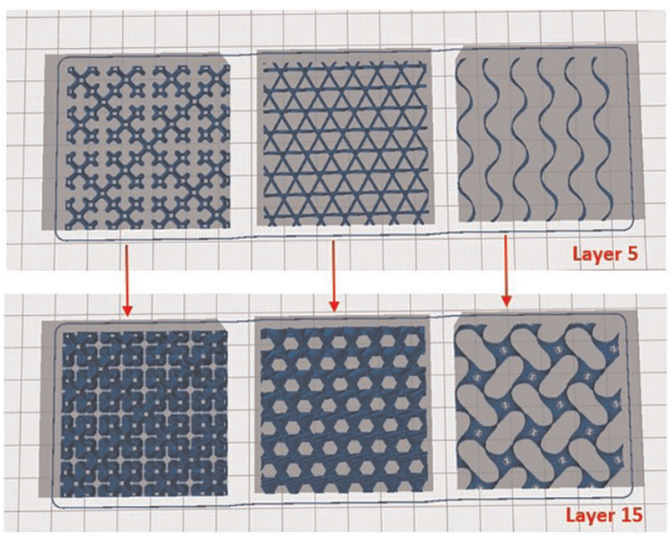

Note: The top set of sliced cuboids are shown at layer 5 and the bottom set are the same cuboids at layer 15 of the print procedure. Pictured from left to right are cross $3 \mathrm{D}$ infill, cubic subdivision infill, and gyroid infill 
materials in various loading states. Recent studies have investigated the ability of the $2 \mathrm{D}$ honeycomb structure to absorb energy in compression (Bates et al., 2016, 2018), characterised 3D printed TPMS-based infill patterns in uniaxial compression (Abueidda et al., 2019; Podroužek et al., 2019) and investigated the ability to print custom shoe-insoles with varying mechanical properties through tailored 3D infill pattern design (Amorim et al., 2019). Podrouzek et al compared the behaviour of different 3D infill patterns in compression (Podroužek et al., 2019), while Wang et al. compared one 3D infill pattern - gyroid infill - to two common 2D infill patterns for energy absorption through the finite element method (Wang et al., 2020). Rossiter et al. (2020) assessed the effects of varying several geometric design variables on the behaviour of FFF nylon samples infilled with truncated octahedron lattices in compression, indicating the importance of infill geometry on the performance of $3 \mathrm{D}$ printed flexible polymers and rubber like materials. A study comparing commercially available 2D and $3 \mathrm{D}$ infill patterns in uniaxial compression as an alternative to VPF in comfort applications, as proposed for this study, has not yet been pursued.

\section{Material and methods}

\subsection{Sample preparation}

\subsubsection{Printing parameters}

A cuboid sample was modelled in Autodesk Inventor Professional 2020 CAD software (Autodesk, Inc., USA) with $25 \mathrm{~mm}$ depth and $50 \mathrm{~mm}$ width and length. This digital model was exported as an .STL file from the CAD software and imported into Ultimaker Cura slicing software version 4.5 (Ultimaker B.V., The Netherlands) for copying and slicing three models per testing group for $3 \mathrm{D}$ printing. The printing parameters used in the slicing software in this study are listed in Table 1. The sample groups and their infill patterns and infill percentages for TPU are shown in Figure 3. The four infill patterns chosen for testing in this study are recommended by Ultimaker to use with flexible filaments, such as the TPU in this study.

Concentric infill has not yet been tested in compression in the literature, though studies show it exhibits lower strengths in tension and flexion than other infill patterns when printed using acrylonitrile butadiene styrene or polyactic acid composites (Khan et al., 2017, 2018; Kesavarma et al., 2020), a characteristic that may be beneficial for comfort applications. Cross infill has also not been tested in the literature; thus, a formal study of its behaviour is beneficial. Cross3D infill and gyroid infill have shown beneficial elastic properties (Abueidda et al., 2019; Pecho et al., 2019), but comparisons to the concentric infill and cross infill have not been conducted in the same flexible material.

The infill percentages chosen were based on previous studies investigating the effects of infill density on performance in compression that showed that infills above $40 \%$ exhibited high strengths (Rodríguez-Panes et al., 2018; Podroužek et al., 2019; Tezel et al., 2019) and the estimated pressures in comfort applications (Apatsidis et al., 2002; Global et al., 2009). Samples were printed on an Ultimaker $2+3 \mathrm{D}$ printer (Ultimaker B.V., The Netherlands) using SpoolWorks FlexD $2.85 \mathrm{~mm}$ green filament (E3D-Online, UK).

3D printed samples were compared to soft SunMate fire retardant grade VPF (Dynamic Systems, Inc, USA). This VPF is used in a variety of applications, including military aircraft seating, wheelchair seating, custom vehicle seating and Formula One vehicle seating. Samples were cut from sheets of $25 \mathrm{~mm}$-thick, $400 \mathrm{~mm}$ by $400 \mathrm{~mm}$ VPF supplied from the manufacturer to measure $50 \mathrm{~mm} \times 50 \mathrm{~mm} \times 25 \mathrm{~mm}(\mathrm{~L} \times \mathrm{W} \times$ $\mathrm{H})$. Three samples were cut to size for compression testing, as set in standard ISO 3386-1 standard for determining the stressstrain characteristics of low-density polymeric materials in compression. The properties of each constituent material tested are listed in Table 2.

\subsubsection{Sample conditioning}

Before testing, all samples were conditioned according to ISO 3386-1. Conditioning consisted of storing samples after preparation for at least $72 \mathrm{~h}$ unloaded at room temperature and relative humidity of $50 \% \pm 5 \%$.

\subsection{Compression testing}

Testing flexible TPU samples was conducted according to ISO $3386-1$. Three specimens were tested for each sample group, as required by ISO $3386-1$ and a $30 \mathrm{kN}$ load cell was used on a Lloyd LR30K Plus Materials Testing Machine (Lloyd Instruments Ltd, England). Data were collected during testing using NEXYGEN MT Materials Testing Software version 4.5.1 Issue 3 (Lloyd Instruments Ltd, England). Before testing, each sample was preconditioned by loading and unloading the sample to $70 \%$ of its initial height three times at a strain rate of $6.67 \times 10^{-4} \mathrm{~s}^{-1}$. Immediately after preconditioning, each sample was again loaded to $70 \%$ of its initial height and then unloaded at a strain rate of $3.33 \times 10^{-3} \mathrm{~s}^{-1}$. All sample data were then averaged for each testing group $(n=3)$. Testing of VPF was conducted following the same procedure with a $5 \mathrm{kN}$ load cell on the same machine, using a smaller load cell due to the lower forces required to compress the VPF to high strains. All data collection was performed at room temperature with a relative environmental humidity of $50 \% \pm 5 \%$.

Table 1 The basic printing parameters used for all thermoplastic polyurethane (TPU) samples

\begin{tabular}{llllllll}
\hline & \multirow{2}{*}{ Layer height } & \multirow{2}{*}{ Top/bottom thickness } & Wall thickness & Print speed & Sample shape & Sample height & Sample width and length \\
\hline TPU & $0.2 \mathrm{~mm}$ & $0.8 \mathrm{~mm}$ & $0 \mathrm{~mm}$ & $30 \mathrm{~mm} / \mathrm{s}$ & cuboid & $25 \mathrm{~mm}$ & $50 \mathrm{~mm}$
\end{tabular}

Note: TPU samples were tested against ISO 3866-1 standard for compressive characteristic of cellular flexible materials. A cuboid sample with the given dimensions was modelled in Autodesk Inventor Professional 2020 CAD software. Printing parameters were set in the slicing software Ultimaker Cura version 4.5. A Omm external wall thickness was set for specimens so that the internal structure was visible during testing, as suggested by the ISO standard 3866-1 
Figure 3 A summary of thermoplastic polyurethane (TPU) sample groups (right) ( $n=24)$, grouped by the dependent variables in this study: infill pattern and infill percentage

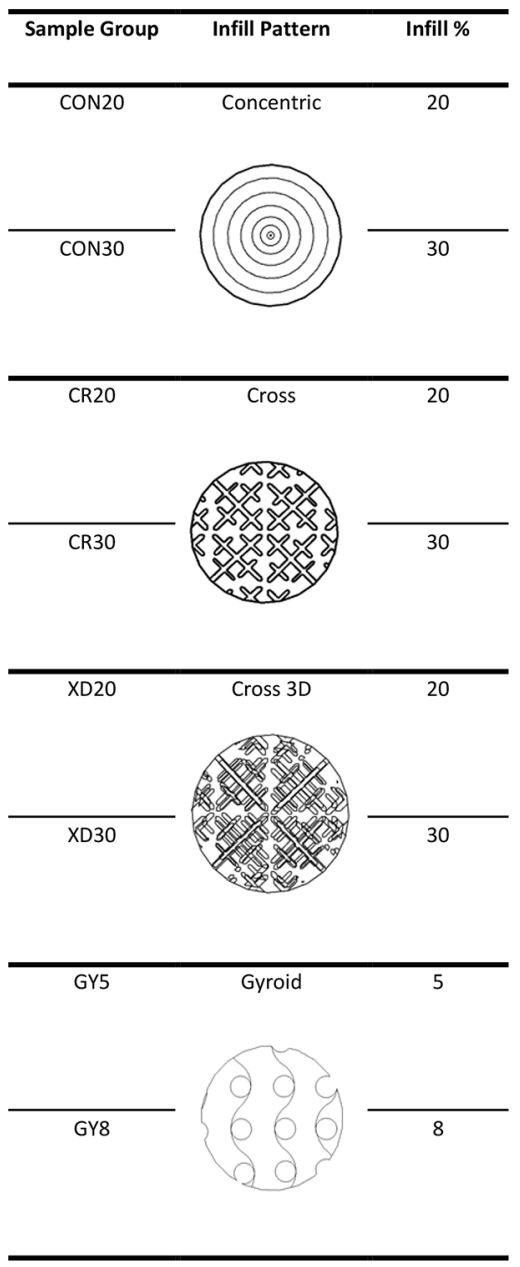

Note: Each TPU sample group consisted of 3 samples, set by ISO 3386-1 standard for compressive characteristic of cellular flexible materials

\section{Results and discussion}

Important material characteristics, as defined in Figures 4 and 5, can be identified from the stress-strain data of flexible VPF and 3D printed TPU. Figure 4 is specific to elastomeric polymers, such as VPF, while Figure 5 is typically seen in elastoplastic materials (Gibson and Ashby, 1997; de Vries, 2009). The important material characteristics for the flexible materials are as follows: the average stiffness in the linear elastic region $\left(E_{i}\right)$; the average collapse stress $\left(\sigma_{c}\right)$ defined as the stress due to which the cells in the polymer structure begin to collapse in compression; the average hysteresis loss at the collapse strain $\left(h_{c}\right)$ of each testing group defined as the average per cent of energy loss in a loading cycle or one minus the ratio of the collapse stress when unloading the specimen $\left(\sigma_{u}\right)$ and the collapse stress when loading the specimen; the plateau stiffness $\left(E_{p l}\right)$ defined as the average stiffness in the plateau region; and the average densification stress $\left(\sigma_{d}\right)$ due to which the cell walls in the polymer structure begin to touch in compression, leading to the structure collapsing fully on itself and a steep increase is seen in the stress-strain data. Note that after the collapse stress in elastoplastic materials, there is a notable drop in the stress before the plateau region begins. This effect is due to the buckling of cell walls in polymer structures with Euler column-like walls

The average characteristic material values for each flexible material testing group from the study results are illustrated in Figures 6-10.

Two-way analysis of variances (ANOVAs) were conducted to assess if the differences between the variances of $E_{i}, \sigma_{c}, h_{c}, E_{p l}$ and $\sigma_{d}$ due to infill pattern, infill percentage and material were statistically significant with $99 \%$ confidence. The results of the two-way ANOVAs and post hoc Tukey honestly significant difference (HSD) tests are summarised in Tables 3-Table 7.

\subsection{Effect of infill percentage}

The compression test stress-strain results for all TPU groups are shown in Figure 11(a)-11(d) for a strain rate of $3.33 \times 10^{-3} \mathrm{~s}^{-1}$. The TPU groups printed with higher infill percentages exhibit higher stiffness, collapse stress, plateau stiffness and densification stress than their lower infill counterparts, as seen in Figures 6-10. However, higher infill also correlates to a lower densification strain, as seen in Figure 11(a)-(d). These effects are expected as there is a higher material mass in the same sample volume. A higher material mass offers more material over which to distribute the compression load, lending strength and stiffness to samples. There is less void space in the structural cells of the higher infill sample groups such that cell walls compress together at a lower strain than in the lower infill sample groups with less material mass. The results here confirm that as infill percentage is increased, the behaviour of the $3 \mathrm{D}$ printed specimen moves towards that of the constituent material, TPU, as found in related literature (de Vries, 2009; Bates et al., 2018). Notably, the behaviour of the concentric infill pattern group changes from elastoplastic at $20 \%$ infill to elastomeric at $30 \%$ infill, though the values in Figure 6-10 suggest that a 30\% infill may be too strong for comfort applications.

Post hoc Tukey HSD tests indicated that infill percentage did not have a statistically significant effect on $E_{i}$ or $h_{c}$ values (Tables 3 and 5, respectively). Its effect on the other three

Table 2 Material properties for the thermoplastic polyurethane (TPU) and viscoelastic polyurethane foam (VPF) tested in this study

\begin{tabular}{lccccc}
\hline Material & Density $\left(\mathbf{k g} / \mathrm{m}^{3}\right)$ & Ultimate tensile strength (MPa) & Young's modulus (MPa) & Elongation at break (\%) & Hardness (Shore A) \\
\hline TPU & 1235 & 42 & 9.8 & 500 & 93 \\
VPF & 80 & 0.131 & $0.122-0.139$ & $137-202$ & -
\end{tabular}

Note: The TPU material is SpoolWorks FlexD green $2.85 \mathrm{~mm}$ diameter filament (E3D-Online, United Kingdom). The VPF is soft fire-retardant grade SunMate foam (Dynamic Systems, Inc., United States). Properties were attained from the respective manufacturers of each material 
Figure 4 Generic stress-strain curve for an elastic material in compression with material characteristics defined

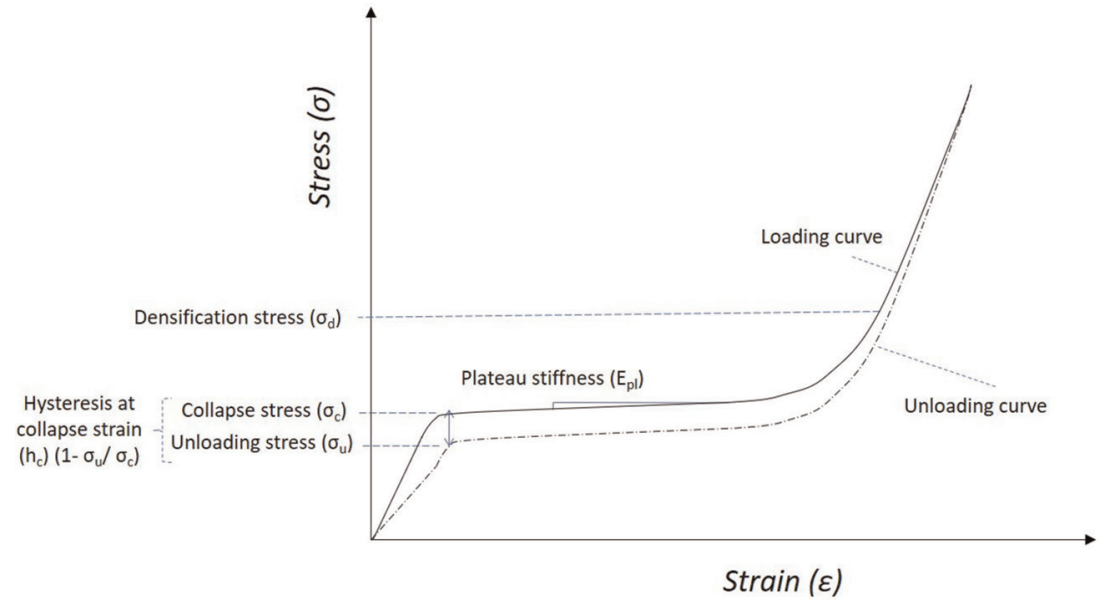

Figure 5 Generic stress-strain curve for an elasto-plastic material in compression with material characteristics defined

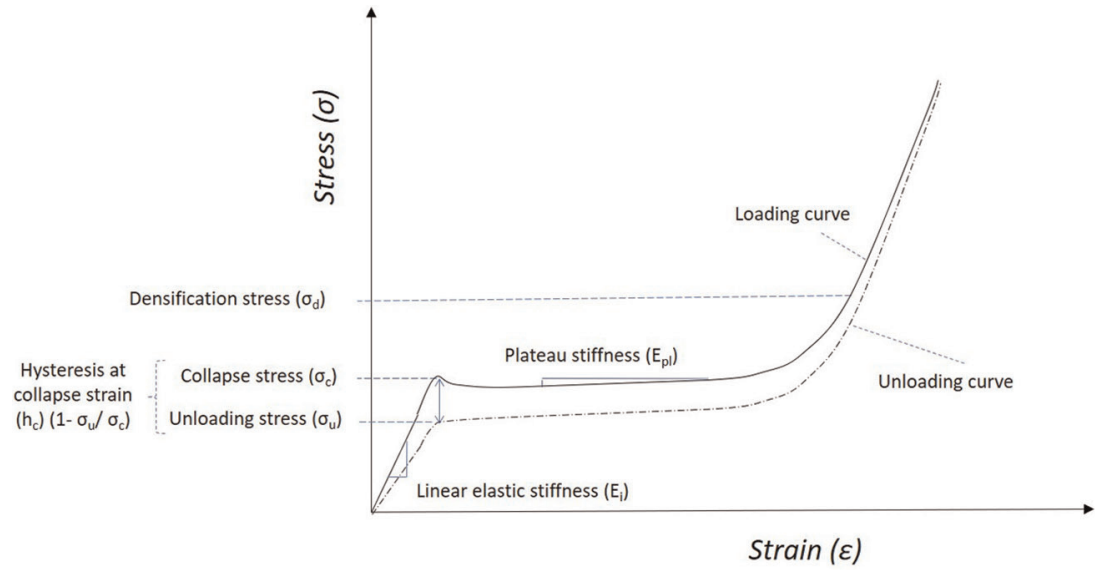

Note: The small decline in stress after the collapse stress which is not present for elastic materials (Figure 4)

Figure 6 The linear elastic stiffness of flexible TPU and VPF sample groups in compression, compared by infill percentage and infill pattern (except foam)

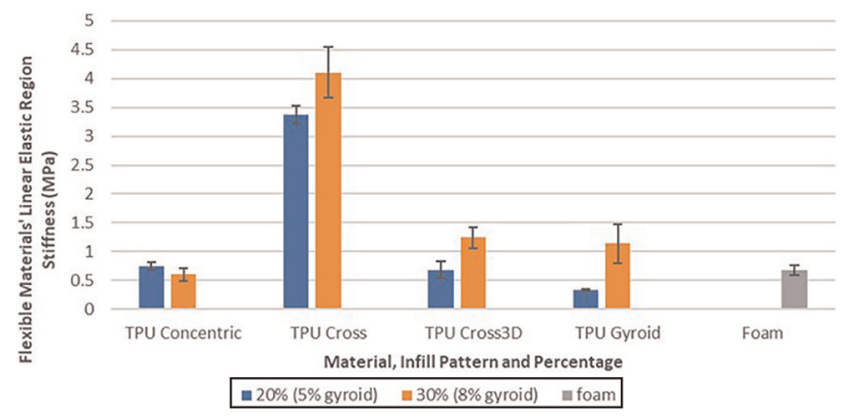

Note: Stiffness was calculated as the Young's modulus in the linear elastic region of each sample's stress strain data and averaged per testing group $(\mathrm{N}=3)$. The standard deviation for each group is shown by the error bars on each column
Figure 7 The collapse stress of flexible TPU and VPF sample groups in compression, compared by infill percentage and infill pattern (except foam)

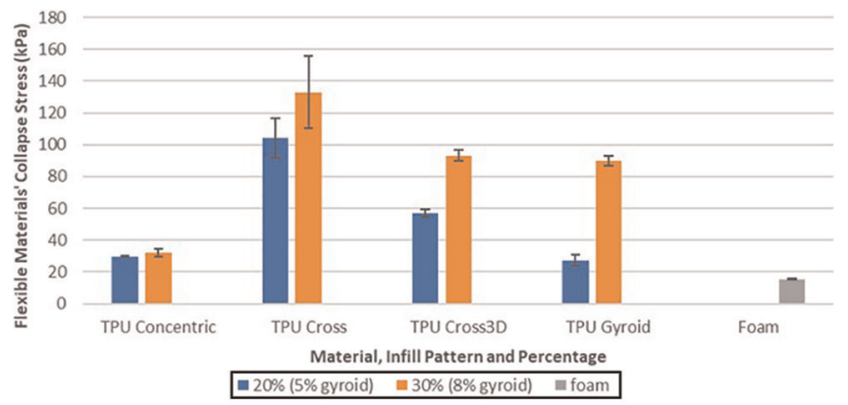

Note: The collapse stress was calculated as the yield stress after the linear elastic region of each sample and averaged per testing group $(\mathrm{N}=3)$. The standard deviation for each group is shown by the error bars on each column 
Figure 8 The average hysteresis loss of flexible TPU and VPF sample groups in compression at the collapse strain $\left(h_{c}\right)$, compared by infill percentage and infill pattern (except foam)

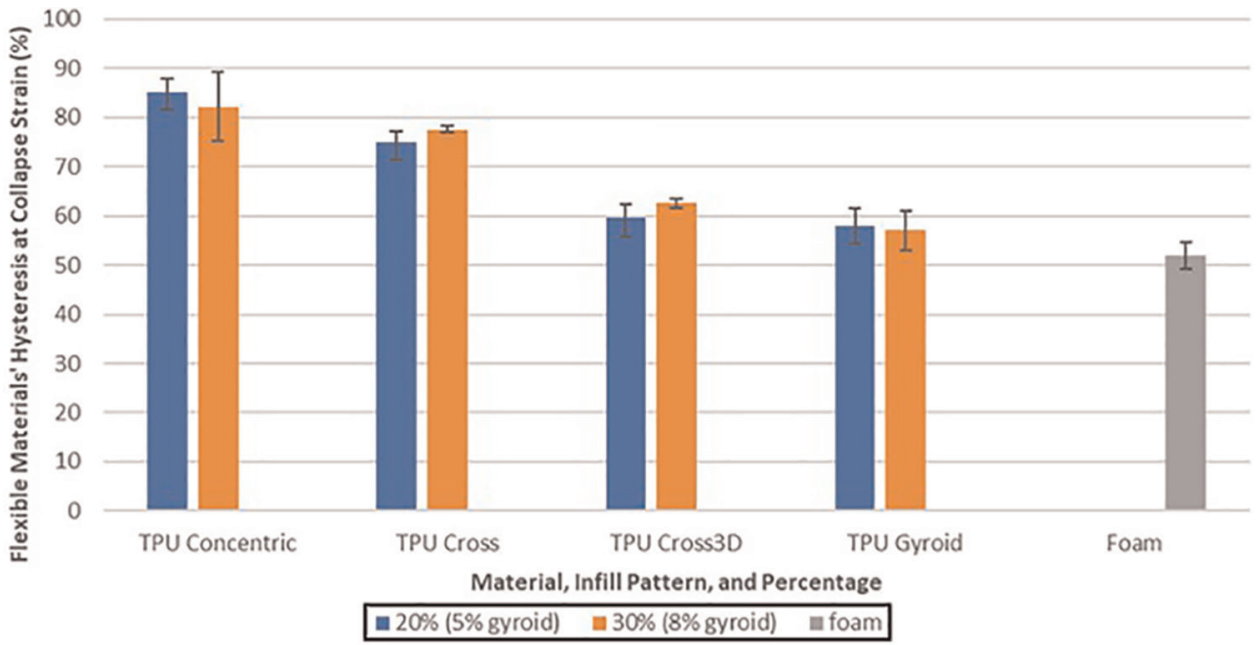

Note: The hysteresis was calculated as (1-(unloading stress/loading stress at collapse)*100) and averaged per testing group $(\mathrm{N}=3)$. The standard deviation for each group is shown by the error bars on each column

Figure 9 The plateau stiffness of flexible TPU and VPF sample groups in compression, compared by infill percentage and infill pattern (except foam)

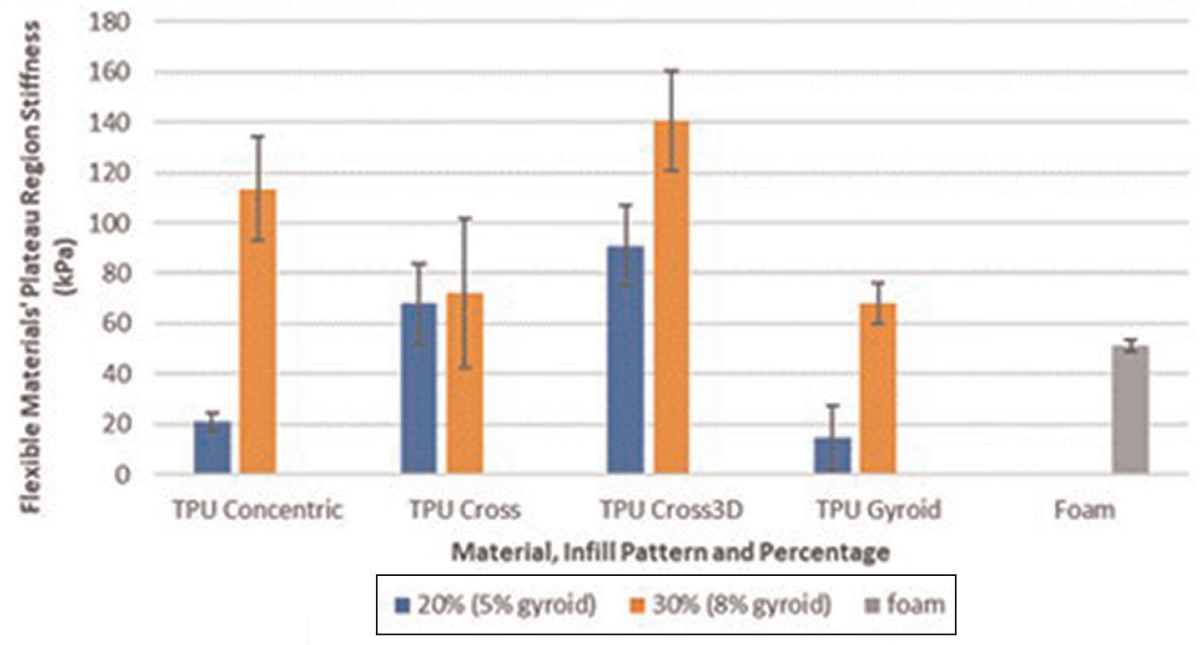

Notes: Stiffness was calculated as the Young's modulus in the plateau region of each sample's stress strain data and averaged per testing group $(n=3)$. The standard deviation for each group is shown by the error bars on each column

material characteristics tends to be a combination interaction with the infill pattern.

\subsection{Effect of infill pattern}

The results in Figures 6-10 suggest that the infill pattern affects all material characteristics defined from the stress-strain data. Figure 6 shows that the cross infill exhibited the highest linear elastic region stiffness $\left(E_{i}\right)$ with the post hoc Tukey HSD test (Table 5) indicating a statistically significant difference between the cross infill pattern groups' $E_{i}$ and those of the other testing groups. The highest collapse stress $\left(\sigma_{c}\right)$ of the flexible sample groups was exhibited by the cross infill pattern groups as seen in Figure 8, with the post hoc Tukey HSD test in Table 4 indicating cross infill's statistically significant different collapse stress from most other groups, somewhat dependent on infill percentage. Figure 8 shows the average hysteresis loss values for all testing groups at their collapse strains. Table 5 summarising the post hoc Tukey's HSD test indicates that the hysteresis loss differed significantly between the 2D infill pattern groups (concentric and cross) and the $3 \mathrm{D}$ infill pattern groups (cross3D and gyroid and VPF with a stochastic, 
Figure 10 The densification stresses of flexible TPU and VPF sample groups in compression, compared by infill percentage and infill pattern (except foam)

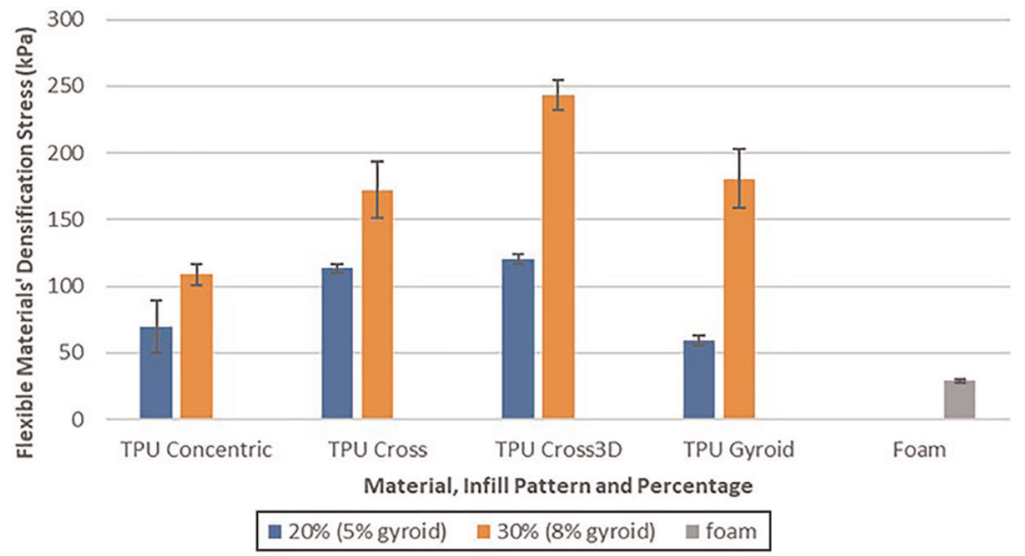

Note: The densification stress was calculated as the stress in each sample's stress strain data after which stress continued to increase quickly and averaged per testing group $(\mathrm{N}=3)$. The standard deviation for each group is shown by the error bars on each column

Table 3 Summary of results of a two-way ANOVA of the linear elastic stiffnesses recorded for sample testing groups, i.e. infill pattern and percentage, in compression at a strain rate of $3.33 \times 10^{-3} \mathrm{~s}^{-1}$

\begin{tabular}{|c|c|c|c|c|c|c|c|c|c|}
\hline Linear elastic stiffness $\left(\mathrm{E}_{\mathrm{i}}\right)$ & Concentric $20 \%$ & Concentric $30 \%$ & Cross $20 \%$ & Cross $30 \%$ & Cross3D 20\% & Cross3D 30\% & Gyroid 5\% & Gyroid 8\% & VPF \\
\hline Concentric $20 \%$ & & & * & * & & & & & \\
\hline Concentric $30 \%$ & & & * & * & & & & & \\
\hline Cross $20 \%$ & * & * & & & * & * & * & * & * \\
\hline Cross $30 \%$ & * & * & & & * & * & * & * & * \\
\hline Cross3D 20\% & & & * & * & & & & & \\
\hline Cross3D $30 \%$ & & & * & * & & & & & \\
\hline Gyroid 5\% & & & * & * & & & & & \\
\hline Gyroid 8\% & & & * & * & & & & & \\
\hline VPF & & & * & * & & & & & \\
\hline
\end{tabular}

Note: An asterisk indicates statistically significant difference $(p<0.01)$

Table 4 Summary of results of a two-way ANOVA of the collapse stresses recorded for sample testing groups, i.e. infill pattern and percentage, in compression at a strain rate of $3.33 \times 10^{-3} \mathrm{~s}^{-1}$

\begin{tabular}{|c|c|c|c|c|c|c|c|c|c|}
\hline Collapse stress $\left(\sigma_{\mathrm{c}}\right)$ & Concentric $20 \%$ & Concentric $30 \%$ & Cross 20\% & Cross $30 \%$ & Cross3D 20\% & Cross3D 30\% & Gyroid 5\% & Gyroid 8\% & VPF \\
\hline Concentric $20 \%$ & & & * & * & & * & & * & \\
\hline Concentric $30 \%$ & & & * & * & & * & & * & \\
\hline Cross $20 \%$ & * & * & & & * & & * & & * \\
\hline Cross $30 \%$ & * & * & & & * & * & * & * & * \\
\hline Cross3D 20\% & & & * & * & & & & & * \\
\hline Cross3D 30\% & * & * & & * & & & * & & * \\
\hline Gyroid 5\% & & & * & * & & * & & * & \\
\hline Gyroid 8\% & * & * & & * & & & * & & * \\
\hline VPF & & & * & * & * & * & & * & \\
\hline
\end{tabular}

Note: An asterisk indicates statistically significant difference $(p<0.01)$

3D cellular structure). Average hysteresis values were lower in the $3 \mathrm{D}$ infill pattern groups, suggesting a $3 \mathrm{D}$ infill pattern in flexible TPU dissipates less energy due to internal friction in compression than $2 \mathrm{D}$ infill patterns.
The cross3D infill pattern groups exhibited the highest plateau region stiffnesses $\left(E_{p l}\right)$, as shown in Figure 9 but the post hoc Tukey HSD test (Table 6) indicated that the effect of the infill pattern on this value varied depending on infill 
Table 5 Summary of results of a two-way ANOVA of the hysteresis loss values recorded for sample testing groups, i.e. infill pattern and percentage, in compression at a strain rate of $3.33 \times 10^{-3} \mathrm{~s}^{-1}$

\begin{tabular}{|c|c|c|c|c|c|c|c|c|c|}
\hline Hysteresis loss $\left(h_{c}\right)$ & Concentric $20 \%$ & Concentric $30 \%$ & Cross $20 \%$ & Cross $30 \%$ & Cross3D 20\% & Cross3D 30\% & Gyroid 5\% & Gyroid 8\% & VPF \\
\hline Concentric $20 \%$ & & & & & * & * & * & * & * \\
\hline Concentric $30 \%$ & & & & & * & * & * & * & * \\
\hline Cross $20 \%$ & & & & & * & * & * & * & * \\
\hline Cross $30 \%$ & & & & & * & * & * & * & * \\
\hline Cross3D 20\% & * & * & * & * & & & & & \\
\hline Cross3D $30 \%$ & * & * & * & * & & & & & \\
\hline Gyroid 5\% & * & * & * & * & & & & & \\
\hline Gyroid 8\% & * & * & * & * & & & & & \\
\hline VPF & * & * & * & * & & & & & \\
\hline
\end{tabular}

Note: An asterisk indicates statistically significant difference $(p<0.01)$

Table 6 Summary of results of a two-way ANOVA of the plateau stiffnesses recorded for sample testing groups, i.e. infill pattern and percentage, in compression at a strain rate of $3.33 \times 10^{-3} \mathrm{~s}^{-1}$

\begin{tabular}{|c|c|c|c|c|c|c|c|c|c|}
\hline Plateau stiffness $\left(\mathrm{E}_{\mathrm{pl}}\right)$ & Concentric $20 \%$ & Concentric $30 \%$ & Cross $20 \%$ & Cross $30 \%$ & Cross3D 20\% & Cross3D $30 \%$ & Gyroid 5\% & Gyroid 8\% & VPF \\
\hline Concentric $20 \%$ & & * & & & & * & & & \\
\hline Concentric $30 \%$ & * & & & & & & * & & \\
\hline Cross $20 \%$ & & & & & & * & & & \\
\hline \multicolumn{10}{|l|}{ Cross $30 \%$} \\
\hline Cross3D 20\% & & & & & & & * & & \\
\hline Cross3D $30 \%$ & * & & * & & & & * & * & * \\
\hline Gyroid 5\% & & * & & & * & * & & & \\
\hline Gyroid 8\% & & & & & & * & & & \\
\hline VPF & & & & & & * & & & \\
\hline
\end{tabular}

Table 7 Summary of results of a two-way ANOVA of the densification stresses recorded for sample testing groups, i.e. infill pattern and percentage, in compression at a strain rate of $3.33 \times 10^{-3} \mathrm{~s}^{-1}$

\begin{tabular}{|c|c|c|c|c|c|c|c|c|c|}
\hline Densification stress $\left(\sigma_{\mathrm{d}}\right)$ & Concentric $20 \%$ & Concentric $30 \%$ & Cross $20 \%$ & Cross $30 \%$ & Cross3D 20\% & Cross3D 30\% & Gyroid 5\% & Gyroid 8\% & $\overline{\text { VPF }}$ \\
\hline Concentric $20 \%$ & & & & * & & * & & * & \\
\hline Concentric $30 \%$ & & & & * & & * & & * & * \\
\hline Cross $20 \%$ & & & & * & & * & & * & * \\
\hline Cross $30 \%$ & * & * & * & & & * & * & & * \\
\hline Cross3D 20\% & & & & & & * & * & * & * \\
\hline Cross3D $30 \%$ & * & * & * & * & * & & * & * & * \\
\hline Gyroid 5\% & & & & * & * & * & & * & \\
\hline Gyroid 8\% & * & * & * & & * & * & * & & * \\
\hline VPF & & * & * & * & * & * & & * & \\
\hline
\end{tabular}

Note: An asterisk indicates statistically significant difference $(p<0.01)$

percentage. For the low-infill testing groups, the average plateau stiffness for the cross3D infill pattern differed significantly from that of gyroid infill. For the high infill groups, the average plateau stiffness for the cross3D infill pattern was significantly different from all other different patterns at a low infill but only significantly different from gyroid at high infill.

Figure 10 shows the average densification stresses $\left(\sigma_{d}\right)$ for each testing group. The post hoc Tukey's HSD test (Table 7) indicated that the effect of infill pattern on $\sigma_{d}$ varied from lowto high-infill percentage. Most low-infill patterns did not behave significantly differently from one another for this material character, except for gyroid and cross3D. For the high-infill percentage groups, the average densification stresses for the cross and gyroid infill patterns were not significantly different from each other and the cross3D infill pattern exhibited average densification stress that was statistically significantly different and higher than all other infill patterns, regardless of infill percentage.

Figure 12 displays the average stress-strain curves for all testing groups including foam in compression at a strain rate of $3.33 \times 10^{-3} \mathrm{~s}^{-1}$. Figures 11 and 12 further suggest that the infill pattern affects the behaviour of the TPU samples in 
Figure 11 Stress-strain data averaged for each 3D printed TPU infill testing group $(n=3)$ at a strain rate of $3.33 \times 10^{-3} \mathrm{~s}^{-1}$

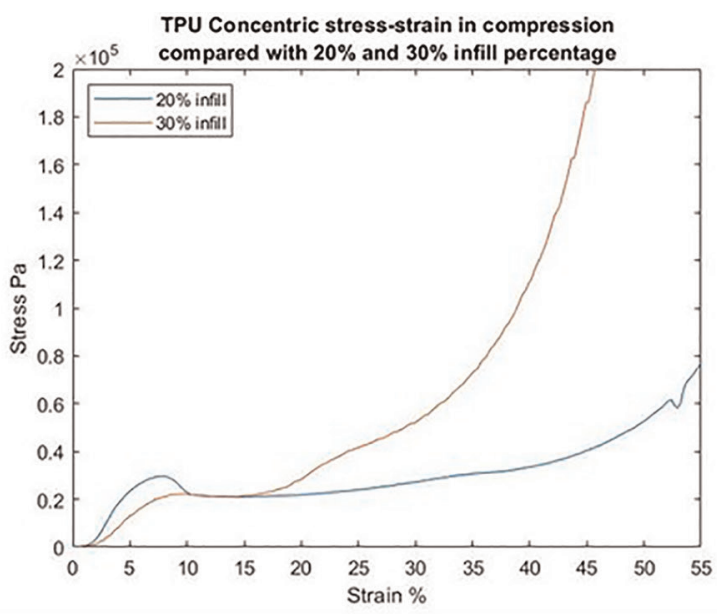

(a)

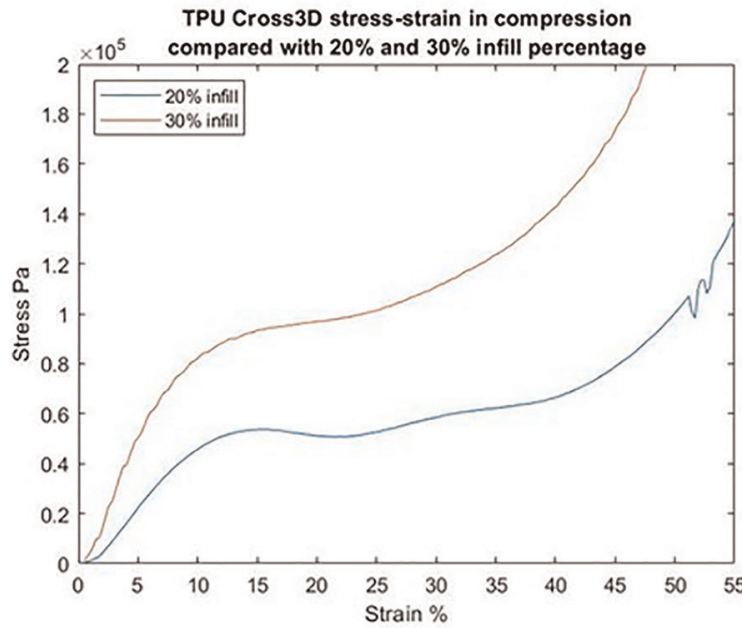

(c)

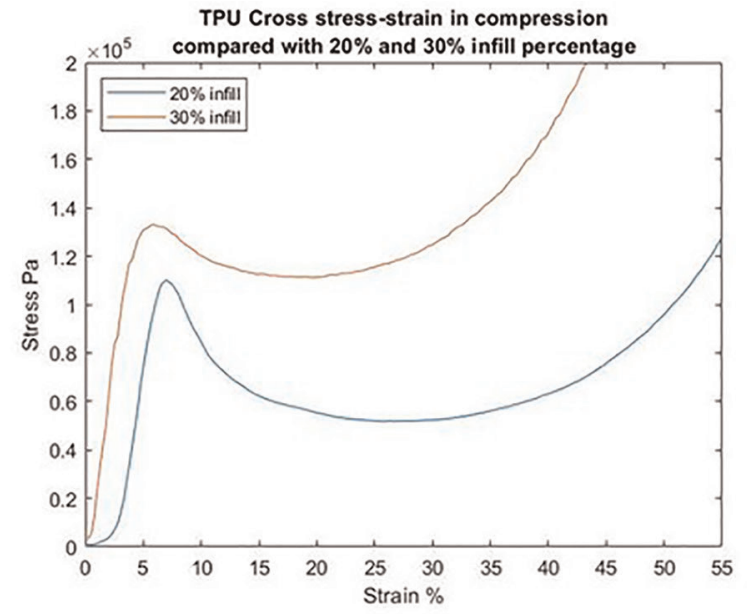

(b)

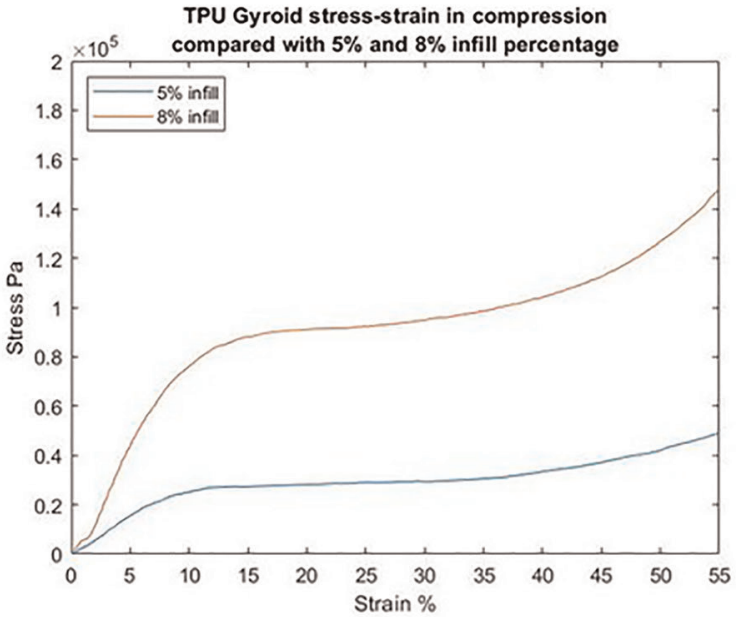

(d)

Notes: Figure 11 -a shows the average stress-strain data for concentric infill with $20 \%$ and $30 \%$ infill percentages. Figure 11-b shows the average stress-strain data for cross infill with $20 \%$ infill and $30 \%$ infill percentages. Figure $11-c$ shows the average stress-strain data for cross $3 \mathrm{D}$ infill with $20 \%$ and $30 \%$ infill percentages. Figure $11-\mathrm{d}$ shows the average stress-strain data for gyroid infill with $5 \%$ and $8 \%$ infill percentages

compression. Concentric and cross infills at low infill percentages exhibit elastoplastic behaviour in compression, such as that shown in Figure 5, while the other infill patterns exhibit behaviour in compression such as that shown in Figure 4 for elastic materials. Elastomeric behaviour is better suited for comfort applications, as an elastoplastic response indicates sudden buckling of the cell structure that may be uncomfortable for the user, translating high levels of vibration or impact to the user. The current results indicate that the internal structure of the 3D printed sample heavily influences its propensity to exhibit either elastomeric (Figure 4) or elastoplastic (Figure 5) response in compression and supports the concept that cell wall structures can be modelled, with simplifications, with Euler column theory, as suggested in related literature on cellular polymers (Gibson and Ashby, 1997; de Vries, 2009).

\subsection{Three-dimensional printed thermoplastic} polyurethane compared to viscoelastic polyurethane foam Results in Figure 6-10 and the statistical analyses in Tables 3-7 suggest that as a lower infill percentage in a $3 \mathrm{D}$ printed TPU sample exhibits a higher strength in compression than VPF, 3D printed infills above $20 \%$ would not be required in comfort applications. It is possible that a lower infill percentage would exhibit stiffness, collapse stress and densification stress closer to VPF, but further investigation is needed to determine the optimum infill percentage range. Printing a TPU material with such a low infill that its stiffness, collapse stress and densification stress are below those of VPF may not be possible, nor advisable due to the possibility of "bottoming-out" or the deformation of a support surface to the extent that pressure redistribution benefits of that would prevent pressure injury development are lost (Ferguson-Pell, 1990; Wang and Dal 
Figure 12 Average stress-strain data ( $n=3$ per testing group) for the different infill groups of TPU compared to viscoelastic foam in compression at a strain rate of $3.33 \times 10^{-3} \mathrm{~s}^{-1}$

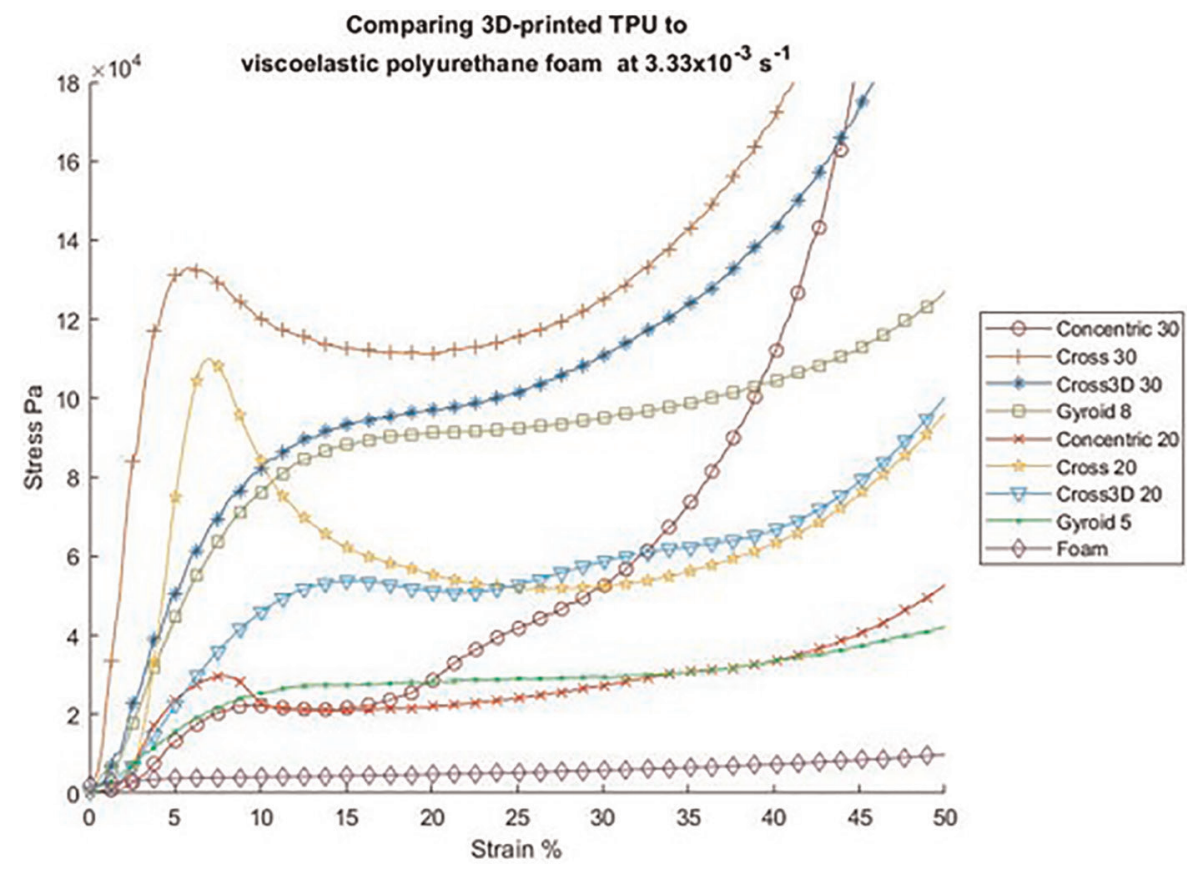

Nevo, 2016; European Pressure Ulcer Advisory Panel, National Pressure Injury Advisory Panel and Pan Pacific Pressure Injury Alliance, 2019).

Figure 12 suggests that the concentric $20 \%$ infill is the nearest to VPF's performance in compression, but the further investigation should be undertaken at a larger scale as Ultimaker Cura slicing software does not have a feature yet for users to define multiple locations from which the concentric pattern grows. The centre is always the point from which the concentric pattern spreads out from, so the pattern concentrates material in the middle of the sliced object. This may not be optimal for VPF comfort applications where pressure redistribution is a key reason for material or manufacturing method choice (Gefen, 2014; Mohanty and Mahapatra, 2014).

The next closest infill pattern in compression behaviour to VPF is gyroid at $5 \%$ infill. As can be seen in Figure 11d, its response is also elastomeric in nature, such as VPF (Briody, 2012). The authors believe this infill pattern would be an effective alternative to VPF in comfort applications due to its 3D structure. The cells in the gyroid samples cause the sample to exhibit stiffness and collapse stress nearer to VPF than the other TPU infill patterns. The gyroid samples have an ordered pattern of cells, while VPF typically exhibits a stochastic structure of different-sized microscopic cells (Briody, 2012). To have a 3D printed support surface behave such as VPF in compression, the authors suggest based on the results of this study that a low-density, 3D infill pattern should be used.

\subsection{Further implications for manufacturing decisions}

The results of this study indicate that a $3 \mathrm{D}$ printed flexible polymer such as the TPU used does not necessarily exhibit similar behaviour to VPF in comfort applications. However, a 3D printed TPU may be a suitable alternative to VPF for products tailored to a specific user, e.g. pilot ejection seats, Formula One car seats, custom wheelchair seating. In the case of custom wheelchair seating, the advantages and disadvantages of producing a custom VPF wheelchair cushion through computer numerically controlled (CNC) milling is compared to those of manufacturing the same cushion in TPU using FFF have been considered in recent literature (Nace et al., 2019). The review concludes that 3D printing may offer a better manufacturing method than CNC milling VPF for custom wheelchair cushions, as $3 \mathrm{D}$ printing enables the creation of complex internal structures in the cushion that help to lower skin temperature and local humidity while keeping manufacturing costs and times similar to those related to CNC milling. These effects, coupled with lowered support pressures resulting from surface contouring in several settings (Tuck et al., 2008; Brienza et al., 2010; Akins et al., 2011; Hosking, 2017), can lower the risk of pressure injury development according to the relevant literature (Gefen, 2011; Jan et al., 2013; Kottner et al., 2018; Tzen et al., 2019).

\subsection{Limitations}

This study used samples sized below $10 \%$ of the volume needed in most support surfaces such as mattresses and seat cushions. Results for larger 3D printed specimens may differ from the samples used in this study and should be investigated in the future. The stress-distribution of a seated model or person is often used to compare the efficacy of different support surfaces (Apatsidis et al., 2002; Akins et al., 2011; McDonald et al., 2015; Bui et al., 2017); the stress distribution on a larger 3D printed support surface should thus be investigated beyond the planar compression investigation conducted here to inform future design and manufacturing decisions. The thermal investigation should also be prioritised to address whether a $3 \mathrm{D}$ 
printed support surface can lower peak pressures while maintaining appropriate temperature and humidity levels to decrease pressure injury risk in the user.

\section{Conclusion}

This study found that both infill percentage and infill pattern have significant effects on the behaviour of $3 \mathrm{D}$ printed TPU in compression. As infill percentage increases, the strength and stiffness of the 3D printed object tend to increase. The effect of the infill pattern is more complex, with different behaviours seen in the infill patterns tested here, regardless of material or infill percentage. Results indicate that of the samples tested, TPU 3D printed at $20 \%$ infill in a concentric pattern behaves most closely to VPF in compression. However, due to concerns related to stress distribution and elastic collapse of infill patterns, the authors recommend a $3 \mathrm{D}$ infill pattern such as gyroid for future development of $3 \mathrm{D}$ printed alternatives to VPF in comfort applications. In the current data set, the gyroid pattern printed at $5 \%$ infill shows promising results. There are numerous different infill patterns available to manufacturers and $3 \mathrm{D}$ printing hobbyists on various slicing programs. This study investigated only four of these patterns in one slicing program, which may not include the optimal infill pattern for comfort applications where VPF is used. The results here are a useful start for further investigation of how different infill patterns affect the behaviour of 3D printed products which are designed for compliance and pressure injury prevention and contributing knowledge to how important infill pattern and density choices are in the design process of any $3 \mathrm{D}$ printed part.

\section{References}

Abueidda, D.W., et al (2019), "Mechanical properties of threedimensional printed polymeric gyroid cellular structures: experimental and finite element study", Materials $\mathcal{E}$ Design, Vol. 165, doi: 10.1016/j.matdes.2019.107597.

Akins, J.S., Karg, P.E. and Brienza, D.M. (2011), "Interface shear and pressure characteristics of wheelchair seat cushions", The fournal of Rehabilitation Research and Development, Vol. 48 No. 3, pp. 225-234, doi: 10.1682/ JRRD.2009.09.0145.

Amorim, D.J.N., Nachtigall, T. and Alonso, M.B. (2019), "Exploring mechanical Meta-material structures through personalised shoe sole design", Proceedings: SCF 2019 ACM Symposium on Computational Fabrication, doi: 10.1145/ 3328939.3329001.

Apatsidis, D.P., Solomonidis, S.E. and Michael, S.M. (2002), "Pressure distribution at the seating interface of custommolded wheelchair seats: effect of various materials", Archives of Physical Medicine and Rehabilitation, Vol. 83 No. 8, pp. 1151-1156, doi: 10.1053/apmr.2002.33987.

Avalle, M., Belingardi, G. and Montanini, R. (2001), "Characterization of polymeric structural foams under compressive impact loading by means of energy-absorption diagram", International fournal of Impact Engineering, Vol. 25 No. 5, pp. 455-472.

Bates, S.R.G., Farrow, I.R. and Trask, R.S. (2016), "threedimensional printed polyurethane honeycombs for repeated tailored energy absorption", Materials \& Design, Vol. 112, pp. 172-183.

Bates, S.R.G., Farrow, I.R. and Trask, R.S. (2018), "Compressive behaviour of three-dimensional printed thermoplastic polyurethane honeycombs with graded densities", Materials \& Design, Vol. 162, pp. 130-142, doi: 10.1016/j.matdes.2018.11.019.

Brienza, D., et al. (2010), "A randomized clinical trial on preventing pressure ulcers with wheelchair seat cushions", Fournal of the American Geriatrics Society, Vol. 58 No. 12, pp. 2308-2314, doi: 10.1111/j.1532-5415.2010.03168.x.

Briody, C. (2012), "The characterisation and numerical modelling of viscoelastic polyurethane foams for use in custom wheelchair seating", doi: 10.21427/D7T60Q.

Bui, T.H., et al (2017),. "Influence of different types of wheelchair cushions for pressure ulcers in view of the experimental approach", Proceedings of the 13th IASTED International Conference on Biomedical Engineering, BioMed 2017, pp. 164-167, doi: 10.2316/P.2017.852-043.

Califano, R., Naddeo, A. and Vink, P. (2017), "The effect of human-mattress interface's temperature on perceived thermal comfort", Applied Ergonomics, Vol. 58, pp. 334-341, doi: 10.1016/j.apergo.2016.07.012.

de Vries, D.V.W.M. (2009), "Characterization of polymeric foams, eindhoven university of technology".

Dealey, C., Posnett, J. and Walker, A. (2012), "The cost of pressure ulcers in the United Kingdom", fournal of Wound Care, Vol. 21 No. 6, pp. 261-266, doi: 10.12968/ jowc.2012.21.6.261.

Domínguez-Rodríguez, G., Ku-Herrera, J.J. and HernándezPérez, A. (2018), "An assessment of the effect of printing orientation, density, and filler pattern on the compressive performance of three-dimensional printed ABS structures by fuse deposition", The International fournal of Advanced Manufacturing Technology, Vol. 95 Nos 5/8, pp. 1685-1695, doi: 10.1007/s00170-017-1314-x.

Dotti, F., et al. (2016), “Thermo-physiological comfort of softshell back protectors under controlled environmental conditions", Applied Ergonomics, Vol. 56, pp. 144-152, doi: 10.1016/j.apergo.2016.04.002.

European Pressure Ulcer Advisory Panel, National Pressure Injury Advisory Panel and Pan Pacific Pressure Injury Alliance (2019), "Prevention and treatment of pressure ulcers/injuries: clinical practice guideline".

Ferguson-Pell, M.W. (1990), "Seat cushion selection", fournal of Rehabilitation Research and Development. Clinical Supplement/Veterans Administration, No. 2, pp. 49-73.

Finn, J.W., et al. (2012), "An integrated child safety seat cooling system-model and test", IEEE Transactions on Vehicular Technology, Vol. 61 No. 5, pp. 1999-2007, doi: 10.1109/TVT.2012.2191427.

Gefen, A. (2011), "How do microclimate factors affect the risk for superficial pressure ulcers: a mathematical modeling study", fournal of Tissue Viability, Vol. 20 No. 3, pp. 81-88, doi: 10.1016/j.jtv.2010.10.002.

Gefen, A. (2014), "Tissue changes in patients following spinal cord injury and implications for wheelchair cushions and tissue loading: a literature review", Ostomy Wound Management, Vol. 60, pp. 34-45. 
Gibson, L.J. and Ashby, M.F. (1997), "Cellular solids structure and properties - Second edition".

Global, S.A.I. et al (2009), "ISO 16840-4: wheelchair seating part 4: seating systems for use in motor vehicles",

Grand View Research Inc (2017), "Pressure relief devices market size, share and trends analysis report by type (lowtech devices, high-tech devices), by region, and segment forecasts, 2018-2025",

Hosking, J. (2017), "A pilot study comparing custom contoured and planar support surfaces for pressure ulcer risk over the heels for night time postural management using interface pressure mapping and discomfort scores", fournal of Tissue Viability, Vol. 26 No. 3, pp. 189-195.

Jan, Y.K., et al. (2013), "Using reactive hyperemia to assess the efficacy of local cooling on reducing sacral skin ischemia under surface pressure in people with spinal cord injury: a preliminary report", Archives of Physical Medicine and Rehabilitation, Vol. 94 No. 10, pp. 1982-1989, doi: 10.1016/ j.apmr.2013.03.022.

Kesavarma, S., et al. (2020), "Bending properties of threedimensional printed coconut wood-PLA composite", IOP Conference Series: Materials Science and Engineering, Vol. 736 No. 5, doi: 10.1088/1757-899X/736/5/052031.

Khan, S.A., et al. (2017), "Evaluation of the effect of infill pattern on mechanical stregnth of additively manufactured specimen", Materials Science Forum, Vol. 887, pp. 128-132, doi: 10.4028/www.scientific.net/MSF.887.128.

Khan, S.F., et al. (2018), "Effect of infill on tensile and flexural strength of three-dimensional printed PLA parts", IOP Conference Series: Materials Science and Engineering, Vol. 429 No. 1, doi: 10.1088/1757-899X/429/1/012101.

Kottner, J., et al. (2018), "Microclimate: a critical review in the context of pressure ulcer prevention", Clinical Biomechanics, Vol. 59, pp. 62-70, doi: 10.1016/j.clinbiomech.2018.09.010.

Kozior, T. and Kundera, C. (2017), "Evaluation of the influence of parameters of FDM technology on the selected mechanical properties of models", Procedia Engineering, Vol. 192, pp. 463-468, doi: 10.1016/j. proeng.2017.06.080.

Kumar, A.R., Fredericks, T.K. and Butt, S.E. (2015), "Using a psychophysical approach to identify a user's self selected thermal comfort on a task chair", International fournal of Industrial Ergonomics, Vol. 46, pp. 36-43, doi: 10.1016/j. ergon.2015.01.005.

Lachenbruch, C. (2005), "Skin cooling surfaces: estimating the importance of limiting skin temperature", Ostomoy Would Management, Vol. 51 No. 2, pp. 70-79.

Lachenbruch, C., et al. (2015), "Relative contributions of interface pressure, shear stress, and temperature on ischemic-induced, skin-reactive hyperemia in healthy volunteers: a repeated measures laboratory study", Wound Management and Prevention, Vol. 61 No. 2, pp. 16-25.

Lin, M., et al. (2019), "three-dimensional-printed flexible polymer stents for potential applications in inoperable esophageal malignancies", Acta Biomaterialia, Vol. 83, pp. 119-129, doi: 10.1016/j.actbio.2018.10.035.

McDonald, R., Sawatzky, B. and Franck, L. (2015), “A comparison of flat and ramped, contoured cushions as adaptive seating interventions for children with neurological disorders", Health Psychology and Behavioral Medicine, Vol. 3 No. 1, pp. 69-81, doi: 10.1080/21642850.2014.981189.

Mohanty, P.P. and Mahapatra, S.S. (2014), “A finite element approach for analyzing the effect of cushion type and thickness on pressure ulcer", International fournal of Industrial Ergonomics, Vol. 44 No. 4, pp. 499-509, doi: 10.1016/j.ergon.2014.03.003.

Nace, S., Tiernan, J. and Ni Annaidh, A. (2019), 'Manufacturing Custom-Contoured Wheelchair Seating: A State of the Art Review', Prosthetics and Orthotics International.

Pecho, P., et al. (2019), "Introduction study of design and layout of UAVs three-dimensional printed wings in relation to optimal lightweight and load distribution", Transportation Research Procedia, Vol. 40, pp. 861-868, doi: 10.1016/j. trpro.2019.07.121.

Podroužek, J., et al. (2019), "Bio-inspired three-dimensional infill patterns for additive manufacturing and structural applications", Materials, Vol. 12 No. 3, pp. 1-12, doi: 10.3390/ma12030499.

Qi, H.J. and Boyce, M.C. (2004), "Stress-Strain behavior of thermoplastic polyurethane", Mechanics of Materials, pp. 1-51.

Rodríguez-Panes, A., Claver, J. and Camacho, A. (2018), “The influence of manufacturing parameters on the mechanical behaviour of PLA and ABS pieces manufactured by FDM: a comparative analysis", Materials, Vol. 11 No. 8, p. 1333, doi: 10.3390/ma11081333.

Rossiter, J.D., Johnson, A.A. and Bingham, G.A. (2019), "Compressive properties of additively manufactured materials compared to foams traditionally used for blunt force trauma protection", in Rapid Design Prototyping $\mathcal{E}$ Manufacturing 16th Conference.

Rossiter, J.D., Johnson, A.A. and Bingham, G.A. (2020), "Assessing the design and compressive performance of material extruded lattice structures", three-dimensional Printing and Additive Manufacturing, Vol. 7 No. 1, pp. 19-27, doi: 10.1089/three-dimensionalp.2019.0030.

Schwartz, D., et al. (2018), "Effects of humidity on skin friction against medical textiles as related to prevention of pressure injuries", International Wound fournal, Vol. 15 No. 6, pp. 866-874, doi: 10.1111/iwj.12937.

Stroupe, K., et al. (2011), "Cost of treating pressure ulcers for veterans with spinal cord injury", Topics in Spinal Cord Injury Rehabilitation, Vol. 16 No. 4, pp. 62-73, doi: 10.1310/ sci1604-62.

Tezel, T., et al. (2019), "Designing and manufacturing of polymer-foam hybrid materials using fused deposition modeling", Advanced Engineering Materials, Vol. 21 No. 6, doi: 10.1002/adem.201800840.

Tsai, K.J., et al. (2017), "Biomimetic heterogenous elastic tissue development", Regenerative Medicine, Vol. 2 No. 1, doi: 10.1038/s41536-017-0021-4.

Tuck, C.J., et al. (2008), "Rapid manufacturing facilitated customization", International fournal of Computer Integrated Manufacturing, Vol. 21 No. 3, pp. 245-258, doi: 10.1080/ 09511920701216238.

Tzen, Y.T., Brienza, D.M. and Karg, P.E. (2019), "Implementing local cooling to increase skin tolerance to ischemia during normal seating in people with spinal cord injury", fournal of Tissue Viability, Vol. 28 No. 4, pp. 173-178, doi: 10.1016/j.jtv.2019.09.006. 
Verstraete, G., et al. (2018), "three-dimensional printing of high drug loaded dosage forms using thermoplastic polyurethanes", International fournal of Pharmaceutics, Vol. 536 No. 1, pp. 318-325, doi: 10.1016/j. ijpharm.2017.12.002.

Wang, Y., et al. (2020), "Numerical and experimental studies on compressive behavior of gyroid lattice cylindrical shells", Materials E Design, Vol. 186, pp. 108340, doi: 10.1016/j. matdes.2019.108340.

Wang, J. and Dal Nevo, R. (2016), “Assessment of memory cushions in aircraft seating for injury mitigation through dynamic impact test", Annual Forum Proceedings - AHS International, Vol. 2, pp. 997-1005, doi: 10.4050/ JAHS.63.012010.

\section{Corresponding author}

Aisling Ni Annaidh can be contacted at: aisling.niannaidh@, ucd.ie 